\title{
Come avviare la terapia nel paziente naïve. Scelta dei farmaci sulle caratteristiche della malattia e del paziente
}

\section{Massimiliano Mirabella}

Responsabile UO Sclerosi Multipla, Fondazione Policlinico Universitario A. Gemelli, Istituto di Neurologia, Università Cattolica del Sacro Cuore, Roma

Nonostante la mancanza di una cura definitiva per la malattia, nell'ultimo decennio si è assistito a un sostanziale incremento delle opzioni terapeutiche per la sclerosi multipla recidivante-remittente (SMRR), associato al progressivo miglioramento di efficacia nel controllo delle ricadute cliniche, talora a costo di un rischio maggiore di tossicità delle cure innovative, che rende necessaria la ricerca di un equilibrio ideale tra efficacia e sicurezza. Un migliore controllo della fase infiammatoria precoce è considerato essenziale per prevenire o ritardare la progressione tardiva, il cui trattamento rimane il vero prossimo

\footnotetext{
Indirizzo per la corrispondenza:

Massimiliano Mirabella

e-mail: massimiliano.mirabella@policlinicogemelli.it

mirabella@rm.unicatt.it

Accettato: 09/12/2015 - Pubblicato online: 18/12/2015

(C) 2015 The Authors. This article is published by HPS Srl and licensed under Creative Commons Attribution-NC-ND 4.0 International (CC BY-NC-ND 4.0). Any commercial use is not permitted and is subject to Publisher's permissions. Full information is available at www.aboutpharma.com/publishing/riviste/aboutopen/
}

grande traguardo da raggiungere nella terapia della SM [1]. I farmaci modificanti il decorso di malattia (disease-modifying therapies, DMT) di più recente introduzione comprendono i farmaci orali dimetilfumarato (DMF) e teriflunomide, che hanno affiancato le terapie immunomodulanti iniettive tradizionali; alemtuzumab, che ha raggiunto altri farmaci di seconda linea come fingolimod e natalizumab, destinati ai pazienti con forme di malattia più attive o non responsive ai trattamenti di prima linea, e le nuove formulazioni di immunomodulanti iniettivi dirette a migliorare l'aderenza alla terapia come PEGinterferone- $\beta 1 \mathrm{a}$ [2] e glatiramer acetato $40 \mathrm{mg}$ [3] (Tabella 1). Tra i nuovi DMT in varie fasi di sperimentazione clinica che nel prossimo futuro si affiancheranno alle terapie in uso vanno ricordati anche daclizumab e ocrelizumab. I DMT attualmente approvati presentano differenze riguardo a meccanismo di azione, efficacia, profilo di sicurezza e tollerabilità, delle quali il clinico deve tenere conto nella selezione del trattamento (Tabella 2). Trattandosi di trattamenti re- 
Tabella 1. Indicazioni dei farmaci attualmente approvati in Italia per la terapia della sclerosi multipla (SM)

\begin{tabular}{|c|c|c|c|c|c|c|c|c|c|c|}
\hline Indicazioni & \begin{tabular}{|c|} 
Interferone \\
beta-1a (Avonex ${ }^{\otimes} /$ \\
Rebif $\left.^{\otimes}\right)$
\end{tabular} & 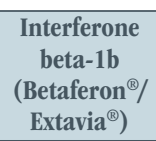 & $\begin{array}{l}\text { Glatiramer } \\
\left.\text { (Copaxone }^{\circledR}\right)\end{array}$ & $\begin{array}{l}\text { Natalizumab } \\
\text { (Tysabri }^{\circledR} \text { ) }\end{array}$ & $\begin{array}{l}\text { Fingolimod } \\
\left(\text { (Gilenya }^{\circledR}\right)\end{array}$ & $\begin{array}{l}\text { Teriflunomide } \\
\left.\text { (Aubagio }^{\circledast}\right)\end{array}$ & $\begin{array}{c}\text { Dimetil- } \\
\text { fumarato } \\
\left.\text { (Tecfidera }{ }^{\circledR}\right)\end{array}$ & $\begin{array}{l}\text { Mitoxantrone } \\
\text { (Novantrone }^{\circledR} \text { ) }\end{array}$ & $\begin{array}{c}\text { Peginterferone } \\
\text { beta-1a } \\
\text { (Plegridy }\end{array}$ & $\begin{array}{l}\text { Alemtuzumab } \\
\left(\text { Lemtrada }^{\circledR}\right)\end{array}$ \\
\hline $\begin{array}{l}\text { Sclerosi multipla recidivante- } \\
\text { remittente (SMRR) }\end{array}$ & Sì & Sì & Sì & & & Sì & Sì & & Sì & $\begin{array}{c}\text { Sì } \\
\text { Malattia } \\
\text { attiva definita } \\
\text { clinicamente } 0 \\
\text { mediante RM }\end{array}$ \\
\hline $\begin{array}{l}\text { Singolo evento } \\
\text { demielinizzante (CIS) con } \\
\text { processo infiammatorio } \\
\text { attivo (gravità tale da rendere } \\
\text { necessario trattamento } \\
\text { con corticosteroidi per via } \\
\text { endovenosa, altre diagnosi } \\
\text { escluse, alto rischio di sviluppo di } \\
\text { SM clinicamente definita) }\end{array}$ & $\begin{array}{c}\text { Sì } \\
\left.\text { (Rebif }^{\circledR} 44 \mu \mathrm{g}\right)\end{array}$ & Sì & Sì & & & & & & & \\
\hline $\begin{array}{l}\text { SM secondaria progressiva } \\
\text { con malattia in fase attiva, } \\
\text { evidenziata da recidive }\end{array}$ & $\begin{array}{l}\text { Avonex }^{\circledR} \text { deve } \\
\text { essere interrotto; } \\
\text { per Rebifi }{ }^{\circledR} \text { non } \\
\text { dimostrata } \\
\text { l'efficacia }\end{array}$ & Sì & Non indicato & Non indicato & Non indicato & \begin{tabular}{c|} 
Non indicato \\
(effetto su recidive \\
e progressione di \\
disabilità in un \\
gruppo di pazienti \\
del TEMSO con \\
elevata attività)
\end{tabular} & $\begin{array}{c}\text { Non indicato } \\
\text { (non testato nelle } \\
\text { forme progressive) } \\
\end{array}$ & \begin{tabular}{|c|} 
Sì \\
In pazienti ancora \\
deambulanti con \\
attività di malattia \\
forma transizionale \\
(rapido \\
peggioramento: uno \\
o più punti EDSS in \\
18 mesi)
\end{tabular} & & \\
\hline \begin{tabular}{|l|} 
Monoterapia SMRR a elevata \\
attività in pazienti che hanno \\
fallito nella risposta a ciclo \\
terapeutico completo con un \\
IFN-beta (o GA nel caso di \\
natalizumab); forma grave a \\
evoluzione rapida (criterio B AIFA)
\end{tabular} & & & & Sì & Sì & & & Sì & & \begin{tabular}{|c|} 
Sì \\
Entrambi i criteri \\
soddisfatti per \\
eleggibilità; non \\
raccomandato \\
in pazienti con \\
malattia inattiva o \\
in quelli stabili con \\
la terapia corrente
\end{tabular} \\
\hline
\end{tabular}

CIS, sindrome clinicamente isolata; EDSS, Expanded Disability Status Scale; GA, glatiramer acetato; IFN, interferone; RM, risonanza magnetica; SMRR, sclerosi multipla recidivante-remittente. 
Tabella 2. Farmaci orali per la terapia della sclerosi multipla recidivante-remittente (SMRR)

\begin{tabular}{|c|c|c|c|c|c|}
\hline Terapia & $\begin{array}{l}\text { Trials } \\
\text { registrativi }\end{array}$ & $\begin{array}{l}\text { Meccanismo } \\
\text { di azione }\end{array}$ & Posologia & $\begin{array}{l}\text { Effetti collaterali } \\
\text { comuni }\end{array}$ & $\begin{array}{l}\text { Effetti collaterali } \\
\text { gravi }\end{array}$ \\
\hline Fingolimod & $\begin{array}{l}\text { TRANSFORMS } \\
\text { FREEDOMS }\end{array}$ & $\begin{array}{l}\text { Ridotta espres- } \\
\text { sione di recettori } \\
\text { S1p1 sui linfociti } \\
\text { con sequestro dei } \\
\text { linfociti nel tessu- } \\
\text { to linfoide. }\end{array}$ & $\begin{array}{l}0,5 \text { mg compres- } \\
\text { se, una cpr/die }\end{array}$ & $\begin{array}{l}\text { Rinofaringite; ce- } \\
\text { falea; fatigue; lin- } \\
\text { fopenia; nausea; } \\
\text { aumento enzimi } \\
\text { epatici; lombal- } \\
\text { gia; diarrea. }\end{array}$ & $\begin{array}{l}\text { Varicella zoster } \\
\text { disseminata; en- } \\
\text { cefalite da herpes } \\
\text { simplex; bradi- } \\
\text { cardia; aritmie } \\
\text { cardiache; bron- } \\
\text { cocostrizione; } \\
\text { edema della ma- } \\
\text { cula; neoplasie } \\
\text { cutanee. }\end{array}$ \\
\hline Teriflunomide & $\begin{array}{l}\text { TEMSO } \\
\text { TENERE }\end{array}$ & $\begin{array}{l}\text { Inibizione della } \\
\text { biosintesi delle } \\
\text { pirimidine nelle } \\
\text { cellule a elevata } \\
\text { attività mitotica. }\end{array}$ & $\begin{array}{l}14 \text { mg compres- } \\
\text { se, una cpr/die }\end{array}$ & $\begin{array}{l}\text { Rinofaringite; di- } \\
\text { sturbi gastrointe- } \\
\text { stinali; lombalgia; } \\
\text { alopecia; innal- } \\
\text { zamento ALT; } \\
\text { cefalea; fatigue; } \\
\text { mialgie arti; infe- } \\
\text { zioni urinarie. }\end{array}$ & $\begin{array}{l}\text { Epatotossicità; } \\
\text { neutropenia; rab- } \\
\text { domiolisi; nevral- } \\
\text { gie, neuropatie; } \\
\text { neoplasie; terato- } \\
\text { genicità. }\end{array}$ \\
\hline Dimetilfumarato & $\begin{array}{l}\text { DEFINE } \\
\text { CONFIRM }\end{array}$ & $\begin{array}{l}\text { Azione su Nrf-2 } \\
\text { (anti-ossidante e } \\
\text { neuroprotettiva). } \\
\text { Riduce citochine } \\
\text { proinfiammatorie } \\
\text { e ingresso di lin- } \\
\text { fociti nel SNC. }\end{array}$ & $\begin{array}{l}240 \text { mg compres- } \\
\text { se, due cpr/die }\end{array}$ & $\begin{array}{l}\text { Flushing; disturbi } \\
\text { gastrointestinali; } \\
\text { cefalea; rinofarin- } \\
\text { gite; fatigue. }\end{array}$ & $\begin{array}{l}\text { Infezioni gravi; } \\
\text { gastroenteriti; } \\
\text { neoplasie (tumori } \\
\text { solidi); gastrite; } \\
\text { linfocitopenia } \\
\text { severa persistente } \\
\text { (PML). }\end{array}$ \\
\hline
\end{tabular}

Cpr, compressa; PML, leucoencefalopatia multifocale progressiva; S1P1, recettore di fingolimod fosfato; SNC, sistema nervoso centrale.

centi, la sorveglianza post-marketing, con particolare attenzione al riconoscimento di effetti collaterali non ancora descritti, è essenziale per confermarne sicurezza ed efficacia a medio-lungo termine. La maggioranza delle evidenze concorda sull'inizio di un trattamento precoce della malattia, ma non esistono a oggi raccomandazioni assolute riguardo la scelta di uno specifico immunomodulante [4]. Pur in assenza di marcatori prognostici assoluti, la severità della malattia può essere valutata tramite i principali elementi clinici e radiologici che influenzano la prognosi (numero di ricadute nei primi 2 anni, intervallo tra le prime due ricadute, grado di recupero e disabilità residua dopo ricaduta, carico lesionale in T2 all'esordio, presenza di lesioni captanti gadolinio) [5-7]. Altri elementi di potenziale significato prognostico - la quantificazione del grado di atrofia e la presenza di lesioni corticali - sono ancora di difficile standardiz- zazione per essere correntemente impiegati in clinica, ma lo diverranno certamente in futuro. Nella pratica clinica i principali elementi che guidano il processo decisionale indirizzando il neurologo nella scelta di uno dei trattamenti DMT nel singolo paziente naïve alla terapia sono:

1) indicatori prognostici del paziente: caratteristiche cliniche e di neuroimaging;

2) profilo di sicurezza del farmaco ed eventuali comorbilità/fattori di rischio che controindichino, in maniera relativa o assoluta, l'utilizzo di uno specifico farmaco nel singolo paziente (Tabella 3);

3) tollerabilità del farmaco e monitoraggio richiesto, che condizionano l'aderenza alla terapia;

4) profilo psicologico e preferenze del paziente, in relazione a età, stile di vita, attività lavorativa, contesto socio-familiare, pianificazione di maternità/paternità; 5) possibile immunogenicità della molecola con po- 


\section{Tabella 3. Comorbilità rilevanti per la scelta dei DMT nel paziente naïve}

- Patologie autoimmuni associate (connettiviti, LES o altre vasculiti): evitare inteferone-beta (IFN- $\beta$ ); utilizzo di immunosoppressori, glatiramer acetato (GA) o impiego di teriflunomide o dimetilfumarato (DMF)

- Distiroidismo/tiroidite autoimmune, grave depressione con ideazioni suicide, epilessia con crisi convulsive non sufficientemente controllate dalla terapia farmacologica (evitare IFN- $\beta$ )

- Ipersensibilità a IFN- $\beta$, GA, albumina, mannitolo, altre allergie note, asma (evitare IFN- $\beta$ o GA per rischio di reazioni di ipersensibilità, broncospasmo, orticaria, anafilassi; in allergici e asmatici cautela anche con DMF)

- Epatopatia/alterazione enzimi epatici (evitare teriflunomide, cautela con IFN- $\beta$, natalizumab, fingolimod)

- Alterata funzionalità renale (cautela con GA)

- Ipertensione arteriosa, diabete e/o neuropatia, assunzione di dicumarolici, anti-diabetici orali, statine, induttori di CYP (cautela con teriflunomide); farmaci che riducono la frequenza cardiaca (cautela con fingolimod)

- Malattia infiammatoria intestinale sintomatica, disturbi gastrointestinali (gastrite, colon irritabile), disturbi del comportamento alimentare (evitare DMF)

CYP, cytochrome P450; DMT, disease modifying therapy; LES, lupus eritematoso sistemico.

tenziale sviluppo di anticorpi neutralizzanti. Un trattamento efficace della malattia richiede l'inizio precoce di un DMT [8], ma deve considerare la futura necessità anche di altre terapie nel lungo termine; dunque, soprattutto per pazienti con esordio di malattia lieve-moderato, è consigliabile un approccio graduale che privilegi la sicurezza prevedendo una "escalation" della terapia in caso di risposta subottimale, identificabile precocemente utilizzando criteri clinici e radiologici. D'altra parte, tale strategia basata sul fallimento di un precedente trattamento e applicata nella maggioranza dei pazienti, in pazienti selezionati con forme più severe di SM potrebbe essere più efficacemente sostituita da una terapia di induzione con DMT più aggressivi [9]. All'inizio del trattamento, alla persona con SM devono essere illustrate le possibili opzioni terapeutiche, indicando qual è la specifica terapia più adatta per la sua malattia, gli obiettivi terapeutici, e istruendo il paziente a riconoscere e gestire i principali effetti collaterali. Minore è l'attività della malattia, maggiore diventa il peso di fattori legati al paziente che influenzano la decisione: età, storia di malattia, genere femmini- le (fertilità, desiderio di gravidanza), accettazione di una cura continua in apparente pieno benessere, uso di farmaci iniettivi (agofobia), necessità di frequenti esami ematochimici di controllo o presenza di effetti collaterali mal tollerati. Ulteriori elementi da considerare nella scelta, potendo modificare l'aderenza al trattamento prescritto, sono la composizione del nucleo familiare (single, figli o familiari a carico), il tipo di attività lavorativa (autonomo/dipendente, lavoro manuale/sedentario, orario fisso/turnista, viaggi e pendolarismo), la presenza di deficit cognitivi o disturbi psichiatrici, che rendono necessaria una supervisione e, in assenza di adeguato supporto, sconsigliano l'utilizzo di farmaci a monitoraggio più complesso.

Esistono anche alcuni criteri oggettivi, incorporati nelle raccomandazioni di molte autorità sanitarie, che guidano il neurologo nella sua decisione:

1) decorso di malattia: tutte le terapie attualmente riconosciute per la SM sono raccomandate per la forma RR, molte per le sindromi clinicamente isolate (CIS), alcune per la fase secondariamente progressiva (SMSP) con ricadute multiple, nessuna per la ma- 
lattia primariamente progressiva (SMPP) [Tabella 1]; 2) durata di malattia: il candidato tipo per il trattamento è la persona con SMRR attiva, ma la cura con farmaci immunomodulanti è sempre più precocemente prescritta al primo episodio clinico. Questa tendenza è maggiore quando la persona con CIS è considerata ad alto rischio di ulteriori attacchi sulla base del quadro clinico e di RM. In effetti è senz'altro razionale il trattamento più precoce possibile di un processo per sua natura cronico e progressivo anche se, in un piccolissimo numero di casi particolarmente benigni, una terapia prescritta potrà risultare aggressiva in rapporto all'entità del quadro clinico e neuroradiologico;

3) attività di malattia: poiché l'intervento terapeutico va modellato sull'individuo, quando nell'anno precedente si è verificato un solo attacco e con un carico lesionale moderato, solitamente si scelgono terapie di prima linea.

Per pazienti con attività clinica e neuroradiologica più elevate (attacchi con disabilità residua, presenza di lesioni tronco-encefaliche e midollari, precoce coinvolgimento cognitivo) si preferisce natalizumab $[10,11] \mathrm{o}$, in alternativa, fingolimod [12] secondo il criterio B di AIFA (SMRR grave a evoluzione rapida, due $O$ più recidive disabilitanti in 1 anno con una $O$ più lesioni captanti-gadolinio alla RM o con un aumento significativo del carico lesionale in $\mathrm{T} 2$ rispetto a una precedente RM). Per i casi più aggressivi di $\mathrm{SM}$, anche in pazienti naïve si dovrebbe considerare il trattamento con alemtuzumab, soprattutto se esiste una controindicazione all'impiego di natalizumab (es. positività per anticorpi anti-JCV con Index elevato) [13]. Il suo complesso profilo di sicurezza (rischio di sviluppo di patologia autoimmune de novo con tireopatia, ITP, nefropatia) ne limita l'indicazione nel paziente nä̈ve (attualmente sulla scheda AIFA di eleggibilità, oltre ai criteri di attività clinica e radiologica, è richiesta la non-responsività a un trattamento precedente), ma è prevedibile un sensibile allargamento dell'impiego di alemtuzumab in questa classe di pazienti, soprattutto in considerazione del suo potenziale ruolo come farmaco di induzione [14, 15]. In passato, in casi di malattia molto attiva, la strategia di induzio- ne, seguita da terapia con DMT, è stata tentata con risultati clinicamente apprezzabili attraverso l'uso di mitoxantrone $[16,17] \mathrm{e}$, in una certa misura, anche di ciclofosfamide, poi progressivamente quasi abbandonati per l'alta incidenza di eventi avversi gravi. Mitoxantrone, per la cardiotossicità e soprattutto il rischio di leucemie indotte dal trattamento $[18,19]$ è oggi esclusivamente utilizzato come farmaco di terza linea nelle forme transizionali (fase precoce della SMSP con rapida progressione clinica e segni radiologici di attività), come anche ciclofosfamide (farmaco AIFA con uso consolidato in neurologia anche se non specificamente approvato per la SM). È prevedibile che i nuovi farmaci orali avranno larga diffusione nei pazienti con SM nä̈ve e la scelta tra i vari farmaci sarà condizionata dai riscontri di efficacia e tollerabilità emergenti dalla pratica clinica [20] Le nuove terapie orali rappresentano un'opzione di trattamento attraente e adeguata in molte situazioni, anche se la scelta di un farmaco orale solo per convenienza di somministrazione non è raccomandabile come pratica generale, data l'assenza di dati completi sulla sicurezza a lungo termine. Inevitabilmente la convenienza di utilizzo e le preferenze del paziente comporteranno una graduale diminuzione nell'impiego di farmaci iniettabili come prima linea di trattamento, a favore dell'incremento dei nuovi farmaci orali, sebbene il profilo di sicurezza (e anche di efficacia in molti pazienti responsivi) a lungo termine di IFN- $\beta$ e GA sia chiaramente favorevole a questi ultimi. Molti pazienti naïve possono iniziare il trattamento con un farmaco iniettabile associato a basso rischio di eventi avversi (Avonex ${ }^{\circledR}$, Betaferon ${ }^{\circledR}$, Copaxone $^{\circledR}$, Rebif ${ }^{\circledR}$ ) anche nelle nuove formulazioni che promettono una migliore aderenza riducendo la frequenza delle somministrazioni (Plegridy ${ }^{\circledR}$, Copaxone $40^{\circledR}$ ). L'uso di fingolimod come farmaco di prima linea in pazienti naïve, a differenza di quanto succede negli Stati Uniti, non è autorizzato in Europa e rimane a oggi controverso [21]. Nel caso di pazienti naïve molto giovani (in cui è prevedibile la necessità futura di un maggior numero di terapie in sequenza), di sesso femminile (possibili gravidanze) o che esprimono timore per possibili effetti a lungo termi- 
ne ancora non conosciuti dei nuovi farmaci orali, agenti come IFN- $\beta$ e GA, anche con i loro effetti collaterali (sintomi simil-influenzali e reazioni locali al sito di iniezione), rimangono il gold standard per l'eccellente profilo di sicurezza a lungo termine e la compatibilità con un'iniziale gravidanza [22]. La prima opzione alternativa è quella di un farmaco orale (Tecfidera ${ }^{\circledR}$, Aubagio ${ }^{\circledR}$ ), il cui uso è spesso percepito dai pazienti come più sicuro di quello che l'assenza di dati a lungo termine imporrebbe. Per alcuni pazienti (agofobici, cefalalgici ecc.) può essere preferibile proporre come terapia iniziale il DMF, anche se la somministrazione due volte al giorno può ridurre la compliance. In questo caso teriflunomide, che si assume in monosomministrazione, può essere un'opzione migliore. Il DMF, in termini di efficacia comparabile se non superiore alle tradizionali terapie iniettive [23, 24], ha un buon profilo di sicurezza, nonostante la necessità di controlli a cadenza trimestrale dell'emocromo per individuare quei rari pazienti che sviluppando linfocitopenia persistente e marcata e possono essere a rischio di sviluppo di leucoencefalopatia multifocale progressiva (PML) [sospendere se linfociti $<500$ o per leucopenia <3000). L'utilizzo di DMF in pazienti naïve può essere limitato dalla scarsa tollerabilità (flushing, sintomi gastroenterici), che si manifesta nei primi mesi in circa il $15-30 \%$ dei pazienti. Se i pazienti vengono adeguatamente istruiti e supportati nei primi mesi per evitare una precoce interruzione del trattamento, il DMF è un efficace farmaco di prima scelta nel paziente naïve [25, 26], anche in considerazione del suo potenziale effetto neuroprotettivo, unico finora tra le terapie disponibili per la SM. Teriflunomide rappresenta un'altrettanto valida opzione in pazienti naïve con attività di malattia lieve o moderata; le pazienti di sesso femminile devono essere informate del rischio in gravidanza e occorre attuare anche una corretta sorveglianza delle possibili complicanze (epatotossicità, neutropenia, ipertensione) mediante l'esecuzione regolare dei controlli previsti ai follow-up e particolarmente frequenti nei primi 6 mesi di terapia [27, 28]. Poiché viene eliminata molto lentamente, è raccomandato inoltre il ricorso alla procedura di eliminazione accelerata in caso di successivo "switch" ad altro DMT. Nei pazienti con CIS, mentre l'uso dei tradizionali DMT iniettivi è indicato sulla base di studi randomizzati controllati, i nuovi farmaci orali non sono ancora formalmente indicati proprio per l'assenza o incompletezza di studi registrativi su questa popolazione di pazienti (Tabella 1). È comunque legittimo attendersi, anche indipendentemente dai trials clinici, un progressivo impiego crescente dei nuovi farmaci orali nei pazienti affetti da CIS, soprattutto quando ad alto rischio di conversione in SM clinicamente definita.

La più importante sfida terapeutica nei pazienti naïve rimane infatti la possibilità di arrestare l'evoluzione della SM con un uso precoce e ottimale dei DMT, singolarmente o in sequenza. Dall'utilizzo di farmaci più potenti è emerso il concetto di "disease-free" e i vari DMT sono comparati in termini della loro abilità soppressiva sull'attività clinica o radiologica della malattia, sebbene non sia ancora chiaro se tale condizione protegga dallo sviluppo di disabilità tardiva. In assenza di studi di comparazione diretta tra farmaci e di biomarcatori affidabili, le analisi di rischio-beneficio generali non consentono di personalizzare la terapia sulla base di evidenze certe. In vista dell'obiettivo di mantenere il paziente libero da ogni attività di malattia andrà valutato anche il potenziale neuroprotettivo dei nuovi farmaci, mentre la farmacogenomica potrà in futuro facilitare la scelta terapeutica attraverso l'identificazione di marcatori di risposta e di rischio a livello individuale. È prevedibile che gli attuali orientamenti terapeutici potranno modificarsi in conseguenza dell'aumentato impiego di farmaci biologici (alemtuzumab, ocrelizumab, daclizumab), dello sviluppo di nuove strategie di trattamento per pazienti con "breakthrough disease" e della migliorata valutazione di efficacia e sicurezza a medio-lungo termine dei nuovi farmaci garantita da trials comparativi e dati di pratica clinica nei pazienti con CIS e SMRR.

\section{Disclosures}

Conflicts of interest: The author declares he has no conflict of interests related to the article. 


\section{Bibliografia}

1. Sorensen PS. New management algorithms in multiple sclerosis. Curr Opin Neurol. 2014;27:246-59.

2. Calabresi PA, Kieseier BC, Arnold DL, et al. Pegylated interferon beta-1a for relapsing-remitting multiple sclerosis (ADVAN$\mathrm{CE})$ : a randomised, phase 3, double-blind study. Lancet Neurol. 2014; 13:657-65.

3. Khan O, Rieckmann P, Boyko A, et al., GALA Study Group. Three times weekly glatiramer acetate in relapsing remitting multiple sclerosis. Ann Neurol. 2013;73(6):705-13.

4. Limmroth V. Treatment of relapsing-remitting multiple sclerosis: current and future algorithms. Eur Neurol. 2014;72:35-8.

5. Tintoré M, Rovira A, Río J, et al. Baseline MRI predicts future attacks and disability in clinically isolated syndromes. Neurology. 2006;67:968-72.

6. Fisniku LK, Brex PA, Altmann DR, et al. Disability and T2 MRI lesions: a 20-year follow-up of patients with relapse onset of multiple sclerosis. Brain. 2008;131:808-17.

7. Confavreux C, Vukusic S, Adeleine P. Early clinical predictors and progression of irreversible disability in multiple sclerosis: an amnesic process. Brain. 2003;126:770-82.

8. Leray E, Yaouanq J, Le Page E, et al. Evidence for a two-stage disability progression in multiple sclerosis. Brain. 2010;133:190013.

9. Gajofatto A, Bacchetti P, Grimes B, et al. Switching first-line disease-modifying therapy after failure: impact on the course of relapsing-remitting multiple sclerosis. Mult Scler. 2009;15:50-8

10. Putzki N, Kollia K, Woods S, et al. Natalizumab is effective as second line therapy in the treatment of relapsing remitting multiple sclerosis. Eur J Neurol. 2009;16:424-6.

11. Hutchinson M, Kappos L, Calabresi PA, et al. The efficacy of natalizumab in patients with relapsing multiple sclerosis: subgroup analyses of AFFIRM and SENTINEL. J Neurol. 2009;256:405-15.

12. Devonshire V, Havrdova E, Radue EW, et al. Relapse and disability outcomes in patients with multiple sclerosis treated with fingolimod: subgroup analyses of the double-blind, randomised, placebo-controlled FREEDOMS study. Lancet Neurol. 2012;11:420-8

13. Cohen JA, Coles AJ, Arnold DL, et al. Alemtuzumab versus interferon beta1a as first-line treatment for patients with relapsing-remitting multiple sclerosis: a randomised controlled phase 3 trial. Lancet. 2012;380:1819-28.

14. Coles AJ, Twyman CL, Arnold DL, et al. Alemtuzumab for patients with relapsing multiple sclerosis after disease-modifying therapy:a randomised controlled phase 3 trial. Lancet. 2012;380:1829-39
15. Menge T, Stuve O, Kieseier BC, Hartung HP. Alemtuzumab: The advantages and challenges of a novel therapy in MS. Neurology. 2014;83:87-97.

16. Edan G, Comi G, Le Page E, et al. Mitoxantrone prior to interferon beta-1b in aggressive relapsing multiple sclerosis: a 3-year randomised trial. J Neurol Neurosurg Psychiatry. 2011;82:134450 .

17. Le Page E, Leray E, Edan G. Long-term safety profile of mitoxantrone in a French cohort of 802 multiple sclerosis patients: a 5-year prospective study. Mult Scler. 2011;17:867-75.

18. Kingwell E, Koch M, Leung B, et al. Cardiotoxicity and other adverse events associated with mitoxantrone treatment for MS. Neurology. 2010;74:1822-6

19. Ellis R, Brown S, Boggild M. Therapy-related acute leukaemia with mitoxantrone: four years on, what is the risk and can it be limited? Mult Scler. 2015;21(5):642-5.

20. Zhang X, Hay JW, Niu X. Cost effectiveness of fingolimod, teriflunomide, dimethylfumarate and intramuscular interferon-beta $1 \mathrm{a}$ in relapsing-remitting multiple sclerosis. CNS Drugs. 2015;29:71-81

21. National Institute for Health and Care Excellence. Multiple sclerosis: management of multiple sclerosis in primary and secondary care. http://www.nice.org.uk/Guidance/CG186. (Accessed December 30, 2014)

22. Cree BA. Update on reproductive safety of current and emerging disease-modifying therapies for multiple sclerosis. Mult Scler. 2013;19:835-43.

23. Gold R, Kappos L, Arnold DL,et al. Placebo-controlled phase 3 study of oral BG-12 for relapsing multiple sclerosis. N Engl Med. 2012;367:1098-107.

24. Fox RJ, Miller DH, Phillips JT, et al. Placebo-controlled phase 3 study of oral BG-12 or glatiramer in multiple sclerosis. N Engl J Med. 2012;367:1087-97

25. Kappos L, Gold R, Arnold DL, et al. Quality of life outcomes with BG-12 (dimethylfumarate) in patients with relapsing-remitting multiple sclerosis: the DEFINE study. Mult Scler. 2014;20:243-52.

26. Kita M, Fox RJ, Phillips JT, et al. Effects of BG-12 (dimethylfumarate) on health-related quality of life in patients with relapsing-remitting multiple sclerosis: findings from the CONFIRM study. Mult Scler. 2014;20:253-7.

27. O'Connor P, Wolinsky JS, Confavreux C, et al. Randomized tria of oral teriflunomide for relapsing multiple sclerosis. N Engl J Med. 2011;365:1293-303

28. Confavreux C, O'Connor P, Comi G, et al. Oral teriflunomide for patients with relapsing multiple sclerosis (TOWER): a randomised, double- blind, placebo-controlled, phase 3 trial. Lancet Neurol. 2014;13:247-56 